\title{
'SHAN-SHUI-HUA' - TRADITIONAL CHINESE LANDSCAPE PAINTING REINTERPRETED AS MOVING DIGITAL VISUALISATION
}

\author{
Christin Bolewski \\ Loughborough School of Art and Design \\ Loughborough University \\ Loughborough, UK, LE11 3TU \\ Leicestershire \\ United Kingdom \\ C.Bolewski@lboro.ac.uk \\ www.lboro.ac.uk
}

\begin{abstract}
This paper is based on a practice-led research project and includes the demonstration of video art. It investigates how East Asian traditions of landscape art can be understood through reference to the condition of Western contemporary visual culture. Proceeding from Chinese thought and aesthetics the traditional concept of landscape painting 'Shan-Shui-Hua' is recreated in modern digital visualisation practice to explore form and question linear perspective that aims to represent realistic space.
\end{abstract}

\section{PERSPECTIVE AND TEMPORALITY IN TRADITIONAL CHINESE LANDSCAPE PAINTING}

The intended purpose and motivation of science and technological progress in our Western civilisation is mainly directed by achieving perfection in measuring and rendering the objective and accountable 'truth' of our world. With regard to the history of the development of visualisation tools this has continuously led towards the invention of hardware and software whose success is measured by its potential to render a more perfect representational image. The creation of real or hyper-real space is one of the main objectives in film and 3D visualisation practice. Based on the Renaissance tradition, achieving the effect of realistic space by the employment of linear perspective is a major preoccupation of Western visual culture. In the construction of a virtual 3D space the Cartesian grid is used to reconstruct this geometrical perspective, whereas Eastern culture has a concept of using multiple vanishing points that within which the creation of a realistic space is not one of its aims. So unlike the Western painting tradition, which under the strong influence of science emphasized proportion, perspective and realistic depiction of form, the Chinese artists never felt compelled to restrict themselves to this limited view. Because a painting is not a window, there is no need to imitate the mechanics of vision and view a scene from only one spot. Instead, they developed a more abstract and free spirited attitude towards art. 'The Chinese artist inspects the world from unrestricted, shifting points of view and is essentially different from the strict and realistic western 'single perspective'. This unique feature enabled Chinese painters to free themselves from the limitations of human vision and permitted more freedom to improvise using ones imagination.' [1]

'And so like a century old guiding principle reads that the painter must have seen a landscape from several viewpoints in order to fathom its being, we find in one and the same picture several perspectives. One and the same figure is not rarely to be seen 
repeatedly in a scroll. We accompany it more or less in its migration. The artists of the traditional Chinese landscape painting do not show us a moment in time, but rather a time span.' [2]

Chinese landscape painting is called 'Shan-Shui-Hua' the three characters standing for respectively mountain, water, and painting. It is composed through a system of multiple vanishing points called 'San-e-ho', the three distances i.e. high distance, deep distance and level distance, also called distance stressing its height, its depth and its leveled broadness. (Many of these Chinese terms are rather ambiguous and not easily explained for our Western minds. They have multiple layers of meaning, with varying interpretations and without exact equivalents in other languages.) This was first established by the painter Kuo Hsi in his famous book 'Sublime notes by forests and rivers': 'Mountain has three distances. Looking at the top of a mountain from its base shows distance stressing its height. Looking at the back of a mountain from its front shows distance stressing its depth. Looking at the mountain beyond from a mountain close by shows distance stressing its leveled broadness.' [3] An artist can combine two or three expressions of distance in a single landscape. The composition of a hanging scroll usually places the foreground at the bottom of the scroll with the middle and far distances moving upward toward the top of the scroll. 'Next to a mountain of great height he might place a valley, creating the illusion of immense depth. Or he might illustrate a panoramic scene with different expressions of space in different parts' [4] 'Early Spring', done in 1072, is the most famous masterpiece by the painter Kuo Hsi and is considered one of the great masterpieces of the Northern Song 'monumental landscape' tradition. [5] (Image can be found at the website of National Palace Museum Taipei) Kuo Hsi arranged the main elements of the painting along the central axis of a vertical scroll. The composition combines the techniques of tall, deep, and flat distance to construct a full-scene landscape.

So multi-perspective as well as temporality are important features for both East Asian aesthetics and the medium of film, and the Chinese horizontal hand scroll is referred to as the first motion picture: it unrolls in time and space and is enjoyed as a progression while the painting is revealed foot by foot. One could say that film montage, specifically 'continuity montage' - the montage of time and space as an addition of shots taken from different viewpoints in a sequence, is assembled in a single picture; the temporal plain is composed within one image. Once the Russian avantgarde filmmaker Sergej Eisenstein was inspired by this commonality and by the Chinese open metaphorical language in the development of his theory of film montage. [6] He studied Japanese language and art and concluded that all the various branches of Japanese art were permeated by the same cinematic element, so that it was appropriate that the cinema should learn from other forms of Japanese artistic practice. Besides extracting techniques from Chinese characters and the Kabuki theatre, Eisenstein had also drawn on traditional scroll paintings that fused in his mind a combined image of close-ups and composition in depth. He was saddened that the montage principles exhibited by the Kabuki plays and the Sino-Japanese ideograms had not been adopted within Far-Eastern cinema, which, in the end had embraced the 'revolting' aesthetic styles of American and European cinema.

\section{'SHAN-SHUI-HUA' REINTERPRETED: THE DIGITAL VIDEO SCROLL}


From the perspective of a contemporary digital media artist and film maker it is very challenging to look once again closer to the relation between East Asian aesthetics and the medium film and to reconsider the early observation of Eisenstein in an approach to digital film practice and to apply it to the condition of digital visualisation technologies and to the constraints that these tools can impose on the structure of video. Confronting the tools of modern computer visualisation with the East Asian concept of the three distances of 'San-e-ho' creates an artistic artefact contrasting, confronting and counterpointing both positions.

My video work 'Shan-Shui-Hua' is not the research of an art historian, it is an individual response as an artist and includes artistic freedom. The contemporary French philosopher Francois Jullien translates Chinese aesthetics for Western understanding. In his book 'The detour over China' (free translation of the German title 'Umweg über China') and other writings. [7] Jullien attempts to create a new approach to Western culture: A 'local change of thinking', a process of distancing from Western thinking as an effective strategy where Chinese philosophy functions as an 'outside' from which to see more clearly the values and preoccupation of Western culture. In the sense of Francois Jullien I make a 'detour over China'. Emphasizing to some extent pictorial concepts and practical aspects of the Chinese painting process enables me to distance and to take a fresh approach to Western film and digital visualisation practice, but at the same time using software tools that generally have been devised to create $2 \mathrm{D}$ and $3 \mathrm{D}$ artefacts from a Western cultural perspective avoids the pitfalls of echoing and imitating Chinese landscape painting to closely. The concept of the three perspectives and the endless scroll are explored through digital filmmaking using the possibilities of $2 \mathrm{D}$ and 3D video compositing and animation tools or virtual camera, depths and particle systems. 

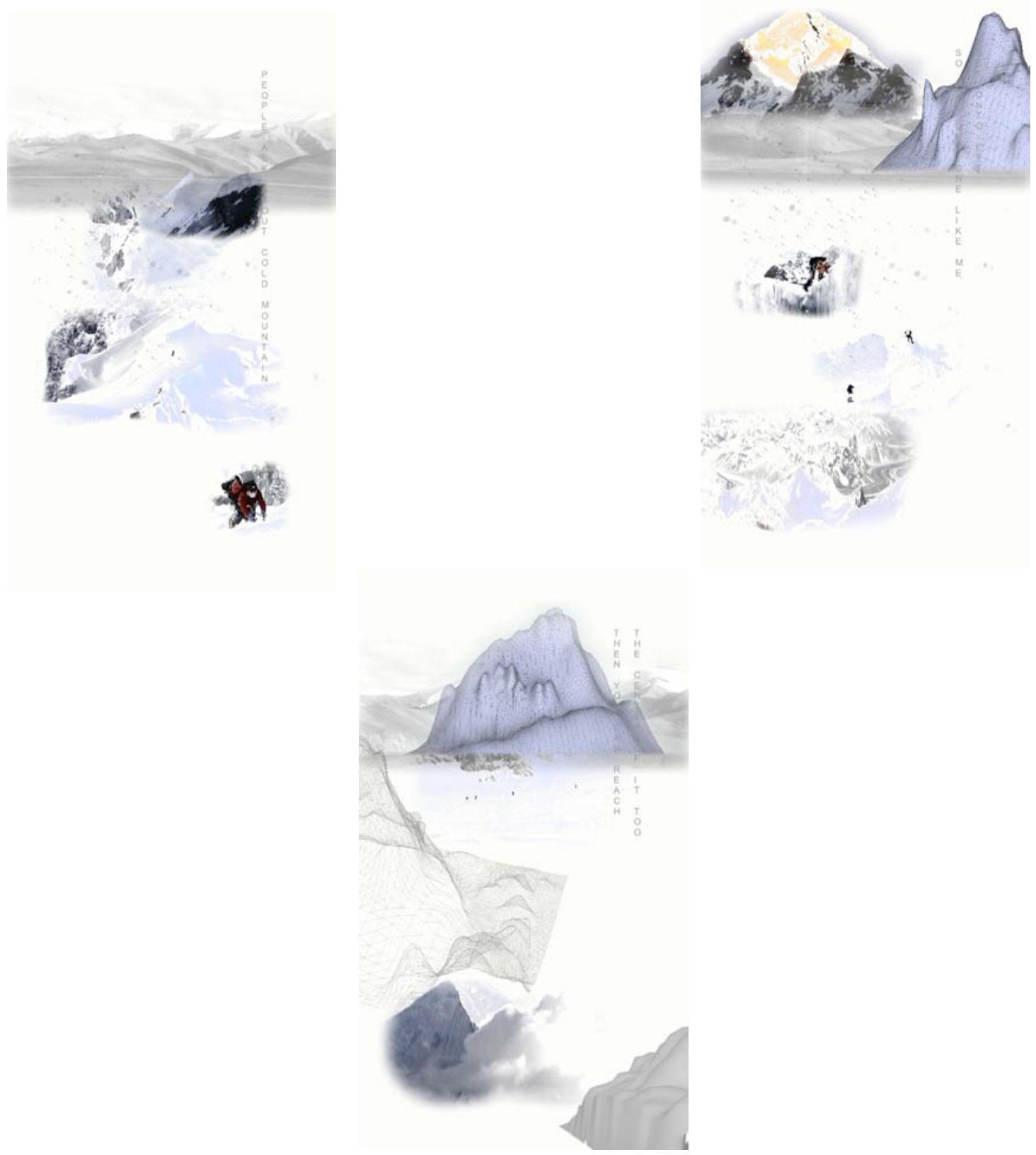

Figure 1-3. 3 video stills from ‘video scroll' Shan-Shui-Hua, (C) Christin Bolewski 2008

My video work 'Shan-Shui-Hua' is a combination of a vertical and horizontal scroll. It is realized in 16:9 High Definition Video providing a detailed high resolution image and is presented on a large flatscreen monitor, wall-mounted in vertical position as a 'video painting' or a 'vertical video scroll'. The horizontal scroll unrolls in time and space, but only in the moment of projection. The video presents a permanent virtual camera movement gliding through an indefinite landscape. This imitates the conception of spatial representation of an original hand scroll where multiple points of view are spread horizontally, parallel to the scene. Whereas in the original hand scroll within the order of space, the order of time introduces a sequence of individual scenes, which gradually unfold, in the video version the order of space can only be introduced in the 
order of time. Off course, the viewer cannot influence the speed in the same manner as it is possible with the 'interactive' traditional hand scroll; this lies within the constraints of the medium film and its inflexible treatment of frame and speed rate. A pre-assigned speed has been set by the artist previously during the creation process and cannot be influenced in the moment of observation. But within the vertical direction the viewer is free to move at $\mathrm{h} / \mathrm{er}$ own speed as in the original vertical scroll. Although the entire scroll can be seen from a distance, a proper appreciation requires close viewing and the space in each section provides a different mode of visual experience accomplished only with up and down eye movements. In the combination of the vertical and horizontal scroll a playful interaction takes place between composition and movement in both directions expanding and combining the possibilities of the two different traditional scroll formats.

The horizontal scroll of the video is composed and animated from right to left in analogy to the East Asian principle. For the Western eye this is an unusual form of composition, because our images are composed with a left to right direction and especially within video and film the movement of approaching or departing objects and camera is determined from left to right. Both cultures also differ in the general treatment of the images. In the Western world, paintings are framed and permanently fixed at a firm place at the wall while Chinese paintings are only enjoyed for occasional viewing pleasures. This corresponds with the non-permanent visibility of film or electronic images only to be observed in the moment of projection or through that consciously turning on of a video monitor. Only the blank screen or projection surface is all time visible and highlights the temporality of the moving image. The 'video scroll' is designed to run endlessly as a permanent looped video sequence without beginning and end, so one could decide to have the work permanently visible on screen serving the Western viewing tradition. The high resolution quality of the HD video format allows to contain numerous small scale objects detailed enough to make satisfying compositions in isolation and enable one intimate scene after another to be revealed and contemplated within the grand scene. Numerous alternatives of lingering interest provide different experiences as within a traditional Chinese painting.

The subject of the Chinese landscape is taken from the material world of threedimensional space, but it exists only in the two-dimensional space of a painting, where volume and depth are illusions. Film / video is a two-dimensional medium as much as painting, but through the addition of the dimension of time movements of objects and camera and therefore the shift of perspective can be captured. This allows the artist to create a simulation of the third dimension of space on the two-dimensional screen. In 3D computer visualisation a simulation of the third dimension can be established through a virtual camera and the creation of 3D objects animated in time. The landscape of the 'video scroll' contains original video recordings as well as 2D / 3D computer generated images and animations of landscapes. Whereas in the original paintings the journey within the third dimension of space is simulated through a careful arrangement of 'San-e-ho' and the repeated appearance of the figure of a solitary traveller (for the viewer to identify with being on a journey) the 'video scroll' additionally uses camera movements along the $\mathrm{x}$ - and $\mathrm{y}$-axis as well as camera zoom and virtual camera movement along the z-axis as an extra layer to push and question the perspective composition of the traditional Chinese painting.

Software for 3D visualisation allows the user to create multiple cameras and viewpoints. Shifting perspective, looking at the scene from different angles and distances can be easily alternated during the process of constructing a virtual space and 
is an important requirement in software design. Most software solutions offer a split screen mode where 4 different perspectives can be monitored simultaneously. During the process of construction the user is free like the Chinese artist to move in space and in his imagination of a landscape, but when it comes to the moment of finally rendering an image or a sequence as a final output there is suddenly only one camera perspective possible at one time. One image can only contain one perspective. The constraints of the software are limited to the laws of linear perspective and the Cartesian Grid. The integration of different viewpoints in one image can only be achieved in a later process i.e. with a compositing tool where different images and perspectives can be composed as a 'collage' through the layering and keying of separate image information. Here it is obvious that the software is designed towards creating artefacts corresponding with 'real' space rather than imaginative space.

In his book 'The Tao of Chinese Landscape Painting' the contemporary artist Wucius Wong creates an interesting analogy to digital visualisation practice: 'But as he paints, the Chinese artist prefers to rely on his own imagination, and arranges the elements as though he were building a model. From his mind-heart he selects mountains, which he can then rotate and reshape; directs water to wind as springs, jump as waterfalls, or flow as rivers along folds; and combines these elements in a harmonious orchestration displaying vital breath... Through acquaintance with the elements, the artist will be able to visualize at will in fabricating his scene. Through understanding of the laws, the artist will position the elements in appropriate positions, directions, and proportions, with transitions, extensions, and overlaps conforming to a definite order... If he wants to have a waterfall, he does not hesitate to put one with the right shape and length at the right place, and to relate it to adjacent elements.' [4] Wucius Wong uses terminology that sounds more familiar describing the construction of a virtual landscape within a digital software application rather then the traditional Chinese painting process. Painting from memory using templates for mountains and waterfalls from a kind of 'construction kit' is strongly reminiscent of the prefabricated models, effects or particle systems of digital software solutions that are ready to be used quickly and only partially adapted for individual use. The 'video scroll' uses templates of mountain models, especially wire frame models, and particle systems for snow, mist or clouds. As the Chinese landscape traditionally does not represent a single landscape rather than an ideal and symbolic form of it, the different elements become a set of 'metaphorical templates' similar as the templates in 3D construction.

In the Chinese landscape as well as in the 'video scroll' different parts of the image sit separately designed within a large empty space. Water, mist, or clouds divide the scene naturally through the interplay of mass and void. Mass and void is an important principle of composition. 'The mist is also empty space, and empty space contains the possibility for transformation. Transformation is central to Taoist thought. It is like being in meditation, when the entire cosmos looks like a white mist, and one finds oneself in a world of white light. Here, time and space seem to be annihilated.' [4] (Zitat suchen) But it also supports the creation of the impression of a continuous landscape although conflicting perspectives are used within the image. The immensity of spatial distance is indicated through vague outlines, through mountain silhouettes disappearing in the fog or through a rather impressionistic treatment of natural phenomenon. The theme of the journey is an enduring theme in landscape paintings. This means yearning for the spiritual, the remote, or the unattainable. The artist frequently portrays himself as a lone figure, a romantic fugitive, wandering into the uninhabited parts of nature where he can linger forever. Chinese landscapes usually include small human figures that 
blend harmoniously into the vast world around them. Man and nature interact and complement each other to reach a state of balance and harmony. The 'video scroll' transposes this traditional relation of man and nature into a different, more contemporary Western manner: it uses the figure of the Western mountaineer equipped with special tools and protective clothing to vanquish the highest peaks in order to conquer nature rather than searching for harmonious existence, thus counterpointing Eastern and Western ideals.

The use of writing that harmonizes with the picture is a further peculiarity of the Chinese painting. The great painters of China were traditionally the same men who were highly educated in the chosen literature. They have long recognized the expressive power of poetry, considering painting as poetry without words. During the Southern Song period (1127-1279), painters were frequently exploiting the connections between poetry and painting, either by making a painting to capture poetic lines or writing a new poem to bring out features of a painting they had done. The 'video scroll' presents two poems of the famous poet Han Shan. [8] He was a hermit and poet of the T'ang Dynasty and most of his poems were written when he lived alone in caves and primitive shelters in the rugged Southern and far Eastern mountains of China. 'Han-shan' means 'Cold Mountain', and part of the delight of his poems is that Cold Mountain is the narrator, the place, and the state of enlightenment that he is trying to tease us into 'getting'. Chinese is a symbol language, ambiguous and open for multiple interpretations. Therefore the poem is open to varying interpretations and within the 'video scroll' it is presented as a reflection on the mountaineers fight against nature ascending and descending the highest peaks counterpointing the Chinese attempt of spiritual harmony.

Once again the French philosopher Francois Jullien translates Chinese aesthetics for Western understanding: in doing so examining and exploring the creation of a meditative space, the tension and harmony between the elements mountain and water, between the compact and fluid, the vertical and horizontal, the white emptiness against fullness, or the bland comprising the harmonious union of all potential values. [9] (Francois Jullien, Das große Bild hat keine Form" German book title) These ideas are inspirational and in opposition to the distinct use of images and the high acceleration of fast moving key visuals overloaded with meaning and message in the current mainstream genres of Western moving image practice. In his film 'Tokyo-ga' made in 1985, as a homage to the Japanese film author Ozu, the German film author Wim Wenders says: 'If only it were possible to make a film like that... Just looking, not trying to prove anything.' [10] Therefore also the 'video scroll' contains no complex narration and attempts to be a meditative open art work - a detour over China combining and contrasting Western and Eastern aesthetics.

\section{References}

[1] ART REALISATION TM: Chinese Traditional Landscape Painting - An Introduction.

http://www.artprintsupply.com/traditional chinese art/landscape painting/intro duction/landscape_painting.htm)

[2] MÜNTER, U and Shuangzhi, L: Formlose Größe. Landschaftsmalerei in der chinesichen Gegenwartskunst. Artnet Magazin 2007. http://www.artnet.de/magazine/features/muenter/muenter01-09-07.asp

[3] WONG, W: The Tao of Chinese Landscape Painting. Principles \& Methods.

New York Design Press. 1991. (English translation of the original text of 
Kuo Hsi)

[4] WONG, W: The Tao of Chinese Landscape Painting, Principles \& Methods. New York Design Press. 1991.

[5] HSI, K: Early Spring. Dated 1072. National Palace Museum, Taipei. Image can be found at

http://www.npm.gov.tw/en/collection/selections_02.htm?docno=48\&catno $=15$

[6] EISENSTEIN, S and Leda, J (ed.): Film form. Harcourt Brace Jovanovich. 1949

[7] JULLIEN, F: Umweg über China. EIn Ortswechsel des Denkens. Merve Verlag Berlin. 2002.

[8] WATSON, B (Translator): Cold Mountain: One Hundred Poems. Columbia University Press. 1970.

[9] JULLIEN, F: Das große Bild hat keine Form. Fink Verlag. 2005.

[10] WENDERS, W: Tokyo-ga. 1985 